\title{
Evaluación de la regeneración natural de tres especies coníferas en áreas de distribución natural en el altiplano occidental de Guatemala
}

\author{
Sergio M. Godínez*, Fabio A. Rodríguez, Nancy P. López, Jorge Camposeco \\ Programa Académico de Ingeniería Forestal, Centro Universitario de Noroccidente, San Marcos,
} Universidad de San Carlos de Guatemala, Guatemala.

\begin{abstract}
*Autor al que se dirige la correspondencia: sergiogodinez_7@yahoo.es
Recibido: 21 de septiembre 2015 / Revisión: 11 de febrero 2016 / Aceptado: 28 de marzo 2016 / Disponible en línea: 01 de agosto 2016
\end{abstract}

\section{Resumen}

$\mathrm{L}$

os bosques de coníferas del altiplano occidental de Guatemala han sido perturbados por intervenciones huma_ nas y fenómenos naturales, lo cual ha propiciado la ocurrencia del fenómeno de regeneración natural. En este escenario se evaluó la densidad de la regeneración natural de tres especies coníferas (Pinus oocarpa Schiede, $P$. pseudostrobus Lindl. y P. tecunumanii Eguiluz \& Perry), en 60 sitios distribuidos en espacios de borde y claro. Las variables evaluadas fueron: fuente de disturbio que originó su establecimiento, variación de la densidad respecto de la fuente de semilla y estimación de la distancia de alcance de dispersión de regeneración adecuada, utilizando unidades de muestreo de $25 \mathrm{~m}^{2}$ para regeneración natural y $500 \mathrm{~m}^{2}$ para los árboles fuente de semilla. Las fuentes de disturbio identificadas fueron: extracción forestal $55 \%$, incendios forestales $24 \%$, plagas $8 \%$, agricultura, alud con $5 \%$ cada una y huracán 3\%. La variación de la densidad de regeneración natural respecto de la fuente de semilla, corresponde a los modelos de J invertida para bordes, y de variable de subpoblaciones para claros. Las distancias de dispersión de regeneración natural establecida para $P$. oocarpa fue $65 \mathrm{~m}$ en borde y $160 \mathrm{~m}$ en claro; para $P$. pseudostrobus fue $75 \mathrm{~m}$ en borde y $175 \mathrm{~m}$ en claro; y para $P$. tecunumanii fue $70 \mathrm{~m}$ en borde y $170 \mathrm{~m}$ en claro. Las distancias de dispersión con densidades aceptables según Instituto Nacional de Bosques-Programa Regional Forestal para Centroamérica, para las tres especies varían de 65-175 m.

Palabras claves: Pinus oocarpa, Pinus pseudostrobus, fuente de disturbio, coníferas, dispersión

\begin{abstract}
$\mathrm{C}$ Coniferous forests of the western highlands of Guatemala have been disturbed by human intervention and natural phenomena, which has allowed the occurrence of the phenomenon of natural regeneration. In this scenario the density of natural regeneration of conifers three species evaluated (Pinus oocarpa Schiede, P. pseudostrobus Lindl. and $P$. tecunumanii Eguiluz \& Perry) in 60 sites distributed in space and clear edge. The variables evaluated were the source of disturbance that caused its establishment, density variation of the source of seed and distance estimation range scattering adequate regeneration, using sampling units of $25 \mathrm{~m} 2$ for natural regeneration and $500 \mathrm{~m} 2 \mathrm{seed}$ source trees. Disturbance sources identified were logging $55 \%, 24 \%$ forest fires, pests $8 \%$, agriculture, avalanche $5 \%$ each and hurricane $3 \%$. The variation of the density of natural regeneration on the seed source, corresponds to the inverted J models for edges, and to clear variable subpopulations. Dispersal distances of natural regeneration was established for P.oocarpa $65 \mathrm{~m}$ in edges, and $160 \mathrm{~m}$ in the clears; P. pseudostrobus $75 \mathrm{~m}$ in edges, and $175 \mathrm{~m}$ in the clears; and P. tecunumanii was $70 \mathrm{~m}$ in edges, and $170 \mathrm{~m}$ in the clears. Dispersal distances with acceptable densities according National Forestry Institute-Regional Forestry Programme for Central American, for the three species vary from $65-175 \mathrm{~m}$.
\end{abstract}

Keywords: Pinus oocarpa, Pinus pseudostrobus, source of disturbance, conifers, dispersion 


\section{Introducción}

La dinámica de los ecosistemas forestales nativos presenta tres fases: el disturbio o perturbación, la invasión o regeneración y la madurez (Rebottaro \& Cabrelli, 2011). White y Pickett (1985) definen el disturbio como "cualquier evento relativamente discreto en el tiempo que trastorna la estructura de una población, comunidad o ecosistema y cambia los recursos, la disponibilidad de sustrato o el ambiente físico"; por su parte, la perturbación se concibe como "un acontecimiento natural o inducido por el hombre, que origina un cambio en las características de los sistemas ecológicos" según Kaufmann y colaboradores (1994); ambos fenómenos generan cambios en la diversidad, estructura y funcionamiento de los ecosistemas. Los disturbios generan claros de diferente magnitud en el dosel, lo que condiciona la cantidad e intensidad de luz que llega al suelo descubierto, factor que define el establecimiento de regeneración natural de especies heliófilas (Chacón, Velásquez, \& Musalem, 1998; González \& Bravo, 1999; Sarasola, Rusch, Schlichter, \& Ghersa, 2006).

El proceso de regeneración natural se define como "la renovación de árboles a través de semillas auto sembradas o por medios vegetativos naturales", según Ford-Robinson citado por Organización Internacional de Maderas Tropicales (2002). También se refiere al proceso por el que en un espacio se produce la aparición de nuevos pies de distintas especies forestales sin la intervención humana y que procede de los pies del arbolado vinculado al área regenerada (Serrada, 2003). La fase de regeneración natural de especies arbóreas forestales es el momento crítico de la vida de cualquier comunidad forestal (González \& Bravo, 1999); le anteceden cuatro momentos: el suministro, la dispersión, la germinación de semillas y la supervivencia (Hawley \& Smith, 1982; Kimmins, 1997). La supervivencia se vincula a la competencia, considerado el factor fundamental en el establecimiento de la regeneración natural de las especies forestales (Oliver \& Larson, 1996).

La formación de la nueva masa forestal en claros dentro del bosque está condicionada por el aporte de semillas de las poblaciones y comunidades ecológicas forestales circundantes (Kimmins, 1997) y los árboles padre constituyen la fuente principal (Rebottaro \& Cabrelli, 2011). La producción de semillas en árboles es directamente proporcional al tamaño del árbol y es afectada por la cantidad de luz disponible para la copa y condiciones climáticas que afectan al crecimiento y reproducción (Greene \& Johnson, 1989; Greene, Messier,
Asselin, \& Fortín, 2002; van Mantgem, Stephenson, \& Keeley, 2006).

La dispersión de semillas se refiere al movimiento de las mismas desde la planta madre y los sitios transitorios de deposición hasta alcanzar su ubicación espacial final, denominada sombra de semillas (Martínez \& González-Taboada, 2009). Se ha establecido que en claros abiertos por incendios de copa o aprovechamiento maderero o desmonte seguidos de una quema, tiende a cubrirse de densa regeneración de Pinos (González \& Bravo, 1999).

Los bosques de coníferas en la región del altiplano occidental de Guatemala, presentan con frecuencia procesos de regeneración natural, principalmente de las especies Pinus oocarpa Schiede, P. pseudostrobus Lindl y P. tecunumanii Eguiluz \& Perry. Estas especies cumplen una función importante en la vida socioeconómica de la población, por los bienes y servicios que producen. En esta región, revisten importancia los departamentos de Huehuetenango, Quiché y Totonicapán, poblados en su mayoría por comunidades indígenas que pertenecen a 10 grupos étnicos, con predominio poblacional las etnias q'iche, mam, q'anjobal y chuj, con índices de desarrollo humano (IDH) de 0.55, 0.50, 0.53 y 0.49 respectivamente, valores considerados bajos e inferiores a la media de IDH nacional estimada en 0.64 . Más del $80 \%$ de estos pobladores se encuentran en condición de pobreza y extrema pobreza (Programa de las Naciones Unidas para el Desarrollo [PNUD], 2014), y que mantienen dependencia de los ecosistemas de bosques de coníferas para satisfacer necesidades de madera y leña para autoconsumo y comercio local.

El contexto enunciado influye en la sobrevivencia de las especies, tal es el caso del $P$. tecunumanii que actualmente está declarada especie protegida de categoría tres según la Lista de Especies Amenazadas de Guatemala (LEA) y especie vulnerable según la Convención Sobre el Comercio Internacional de Especies Amenazadas de Flora y Fauna Silvestres (Cites) (Consejo Nacional de Áreas Protegidas, 2009). En el manejo forestal tradicional de Guatemala, la regeneración natural como opción ecológica y económica para la restauración forestal de áreas disturbadas ha sido de poca importancia; a pesar de sus ventajas biológicas, ecológicas y económicas (Rebottaro \& Cabrelli, 2011). Algunas de las ventajas que pueden mencionarse son: la diversidad genética, individuos adaptados y seleccionados naturalmente y los costos de establecimiento que se limitan únicamente a los de oportunidad. A cambio de ello se promueven proyectos basados en actividades 
de producción de plántulas en viveros y establecimiento de plantaciones forestales de especies coníferas, con la consecuente disminución o empobrecimiento de la riqueza genética.

El objetivo general del estudio fue determinar la capacidad regenerativa de pino colorado (P. oocarpa), pino triste (P. pseudostrobus) y pino ocote (P. tecunumanii); y los específicos, identificar la fuente de disturbio que ha originado el establecimiento de áreas con regeneración natural, determinar la variación de la densidad de la regeneración natural respecto a la distancia de la fuente de semilla o progenitores, y estimar el alcance de dispersión que determine el establecimiento de una regeneración adecuada, en el altiplano occidental de Guatemala.

Las mayores densidades de regeneración natural en número de individuos/ha, se registraron en sitios afectados por alud con P. oocarpa con 11,773; luego en agricultura con P. pseudostrobus con 5,570; finalmente con $P$. tecunumanii en áreas de aprovechamiento forestal con 5,500 e incendios forestales con 4,566. Las mayores densidades por disturbio en número de individuos/ha, de especies asociadas fue de 19,170 en incendio forestal, de 15,908 en aprovechamiento forestal, y de 13,394 en agricultura, las tres asociadas a P. pseudostrobus.

Los modelos de variación de la densidad de regeneración natural respecto de la fuente de semilla, fueron $\mathrm{J}$ invertida para bordes y subpoblaciones variables para claros. Las distancias de dispersión variaron entre 90 a $115 \mathrm{~m}$ en bordes y entre 160 a $190 \mathrm{~m}$ en claros. La exposición norte favoreció a las tres especies con las mayores densidades, que varían entre 7,864 a 5,378 individuos/ha.

\section{Materiales y métodos}

El estudio se realizó en las zonas de vida bosque húmedo montano subtropical, bosque muy húmedo montano bajo subtropical, bosque húmedo subtropical templado según el sistema Holdridge, dentro del territorio de los departamentos de Huehuetenango, Quiché y Totonicapán (Figura 1).

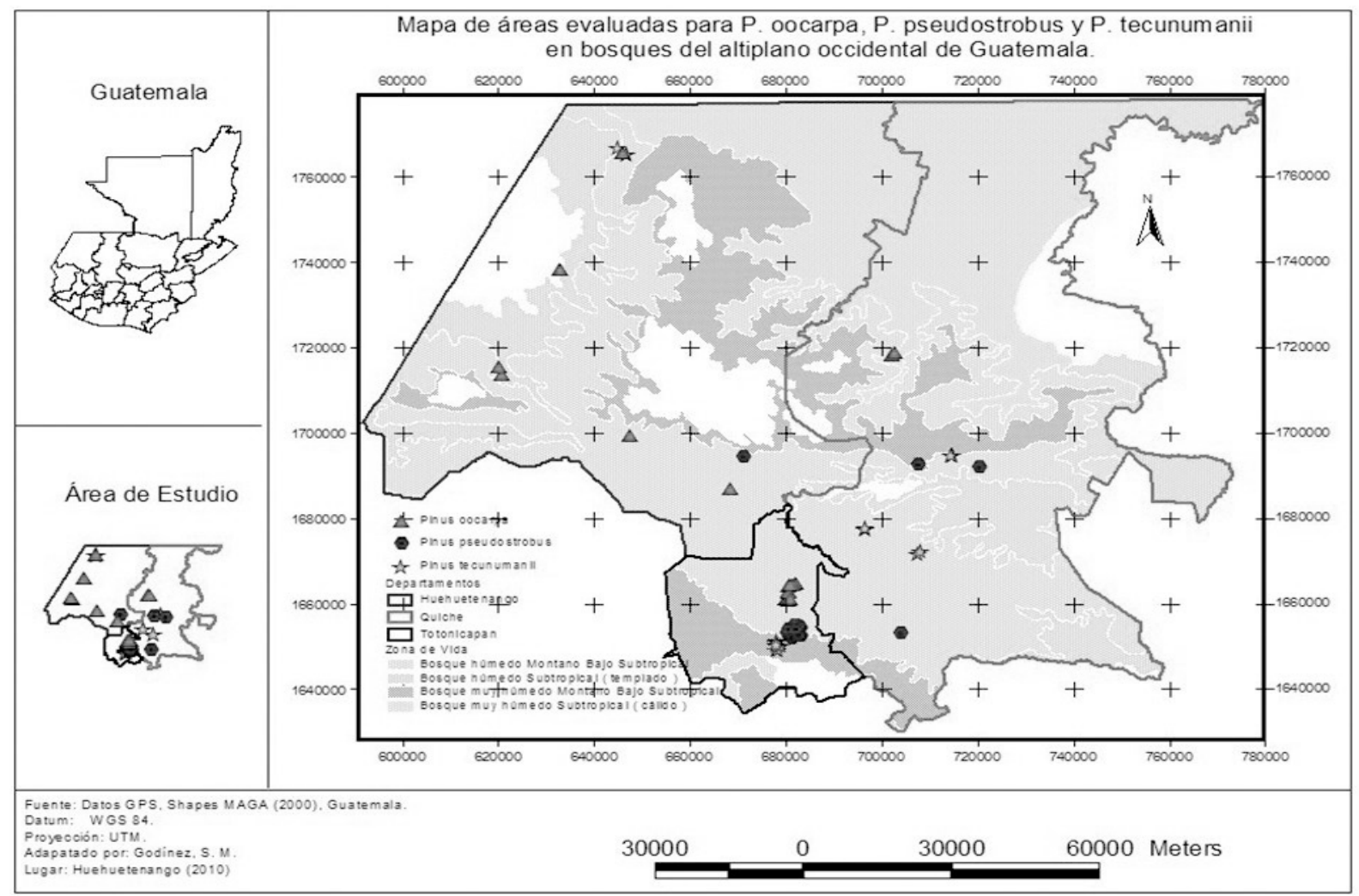

Figura 1. Mapa de zonas de vida y distribución de sitios de evaluación de regeneración natural de Pinus pseudostrobus, P. tecunumanii, P. oocarpa, en los departamentos de Huehuetenango, Quiché y Totonicapán. 
La colecta de datos en terreno se realizó de febrero a noviembre de 2009. El área se estratificó en función de las zonas de vida como primera etapa, y a lo interno de éstas se seleccionaron sitios con regeneración natural de pinos en espacio de bordes y claros como segunda etapa de estratificación. Para cada espacio de regeneración, se seleccionaron dos sitios de evaluación para cada exposición fisiográfica por especie (Tabla 1). El número de sitios evaluados se definió en función de la temporalidad del estudio, el área geográfica y el número mínimo de sitios regenerados por especie por clase de disturbio, hallado y de los que se obtuvieron autorización de los propietarios para estudiarlos.
En cada sitio de regeneración natural se trazaron unidades de muestreo (UM) de $500 \mathrm{~m}^{2}$ para evaluar la masa boscosa madura posible fuente de semilla y UM continuas de $25 \mathrm{~m}^{2}$ para evaluar la regeneración natural. El número de las UM continuas fue variable, en función de la distancia hasta donde existían plántulas regeneradas, para cumplir el propósito de determinar el alcance de dispersión de la regeneración. La orientación de las UM continuas y de evaluación de los árboles fuente de semilla, fue perpendicular al borde dominante del bosque para espacios de borde y en dirección a los cuatro puntos cardinales para espacios de claro (Figura 2).

Las variables evaluadas se relacionan con el arbolado adulto y la regeneración (Tabla 2), así como la

Tabla 1

Distribución de sitios de regeneración evaluados

\begin{tabular}{|c|c|c|c|c|c|}
\hline \multirow{2}{*}{$\begin{array}{l}\text { Espacio de } \\
\text { regeneración }\end{array}$} & \multirow{2}{*}{$\begin{array}{l}\text { Exposición } \\
\text { Fisiográfica }\end{array}$} & \multicolumn{3}{|c|}{ Sitios evaluados/especie } & \multirow{2}{*}{ Total } \\
\hline & & Pinus oocarpa & Pinus pseudostrobus & Pinus tecunumanii & \\
\hline \multirow[t]{5}{*}{ Borde } & Norte & 2 & 2 & 2 & 6 \\
\hline & Sur & 2 & 2 & 2 & 6 \\
\hline & Este & 2 & 2 & 2 & 6 \\
\hline & Oeste & 2 & 2 & 2 & 6 \\
\hline & Cenital & 2 & 2 & 2 & 6 \\
\hline \multirow[t]{5}{*}{ Claro } & Norte & 2 & 2 & 2 & 6 \\
\hline & Sur & 2 & 2 & 2 & 6 \\
\hline & Este & 2 & 2 & 2 & 6 \\
\hline & Oeste & 2 & 2 & 2 & 6 \\
\hline & Cenital & 2 & 2 & 2 & 6 \\
\hline Total & & 20 & 20 & 20 & 60 \\
\hline
\end{tabular}

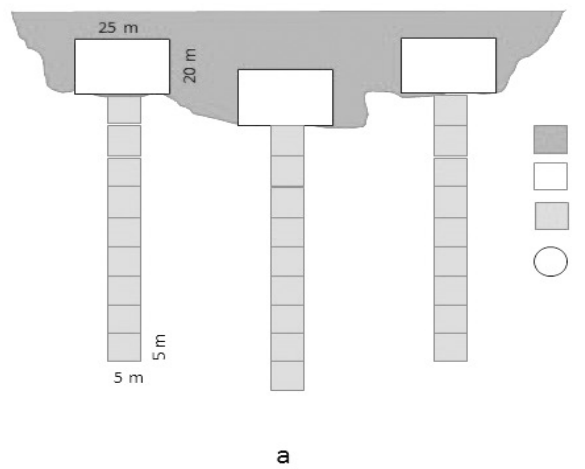

Bosque maduro

UM $500 \mathrm{~m}^{2}$

UM $25 \mathrm{~m}^{2}$

Claro de bosque

UM: Unidad de muestreo

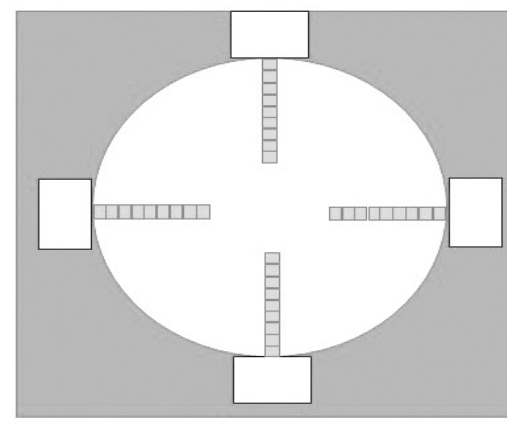

b

Figura 2. Modelos de evaluación de arbolado progenitor y regeneración natural de pinos en espacios de (a) borde de bosque y (b) claros de bosque. Diseño metodológico y gráfico: Sergio Miguel Godínez. 
fuente de disturbio. Las especies asociadas a la regeneración evaluadas fueron arbustos y herbáceas; para el arbolado adulto, la calidad del fuste evaluado se clasificó en recto, inclinado, sinuoso y bifurcado; la calidad de árbol semillero en excelente, bueno e indeseable; el daño biológico en ataque de insectos, de hongos y podrido, y el daño mecánico por ocoteo, desramado y fuste quemado.

La estimación de la densidad de regeneración, se realizó transformado la densidad hallada en cada unidad de $25 \mathrm{~m}^{2}$ a su equivalente en hectáreas por medio de la ecuación 1 (Godínez, Rodríguez, Camposeco, \& López, 2010).

$$
\frac{\text { (Ecuación 1) }}{h a}=\left[\frac{10000 \frac{m^{2}}{h a}}{25 \frac{m^{2}}{U M}}\right] /\left[\frac{\text { Individuos }}{U M}\right]
$$

El valor hallado de densidad, se asignó a la respectiva UM continua de $25 \mathrm{~m}^{2}$, dato utilizado para la estimación de la densidad a la distancia de ubicación de la UM continua.
Para la estimación de una regeneración adecuada o establecida se usó el criterio de $\geq 900$ individuos/ha, definido por el Instituto Nacional de Bosques (Inab) y el Programa Regional Forestal para Centroamérica (Procafor) (Inab, 2001).

\section{Resultados}

Las fuentes de disturbio que antecedieron al establecimiento de la regeneración natural en los 60 sitios evaluados, 20 por especie, fueron seis (Figura 3), citados en orden de importancia: aprovechamientos forestales $(55 \%)$, incendios forestales (24\%), plagas (Dendroctonus sp.) (8\%), agricultura y alud con $5 \%$ cada una y huracán (Mitch) 3\%. Las categorías de desarrollo evaluadas como regeneración natural corresponden a plántula, brinzal y latizal.

En sitios afectados por aprovechamiento e incendio forestal se halló regeneración de las tres especies. En sitios afectados por agricultura se encontró regeneración de $P$. pseudostrobus y P. tecunumanii, únicamente. En los afectados por plagas forestales se encontró únicamente regeneración de $P$. tecunumanii. En los sitios

Tabla 2

\begin{tabular}{|c|c|c|c|}
\hline $\begin{array}{l}\text { Categoría de } \\
\text { desarrollo }\end{array}$ & $\begin{array}{l}\text { Criterios de clasificación de las categorías } \\
\text { de desarrollo }\end{array}$ & Variables evaluadas & $\begin{array}{l}\text { Unidad de } \\
\text { muestreo }\end{array}$ \\
\hline Plántula & Individuos $<30 \mathrm{~cm}$ de altura & \multirow{3}{*}{$\begin{array}{l}\text { Nombre de la especie } \\
\text { Número de individuos } \\
\text { Especies asociadas }\end{array}$} & \multirow{3}{*}{$\begin{array}{c}25 \mathrm{~m}^{2} \\
(5 \times 5 \mathrm{~m})\end{array}$} \\
\hline Brinzal & $\begin{array}{l}\text { Individuos de } 0,30 \mathrm{~m} \text { hasta } 1,50 \mathrm{~m} \text { de altura } \\
\qquad y \text { dap }<5 \mathrm{~cm} \\
\text { Individuos de } 5,0 \text { a } 9,9 \mathrm{~cm} \text { de dap }\end{array}$ & & \\
\hline Fustal & Individuos a partir de 10 a $25 \mathrm{~cm}$ de dap & & \\
\hline Árbol maduro & Individuos con dap $>$ de $25 \mathrm{~cm}$ & $\begin{array}{l}\text { Calidad de fuste } \\
\text { Clase de semillero } \\
\text { Daño biológico } \\
\text { Daño mecánico } \\
\text { Dap en cm. y } \\
\text { Altura en m }\end{array}$ & $\begin{array}{c}500 \mathrm{~m}^{2} \\
(20 \times 25 \mathrm{~m})\end{array}$ \\
\hline
\end{tabular}

Variables evaluadas en regeneración natural y arbolado maduro 


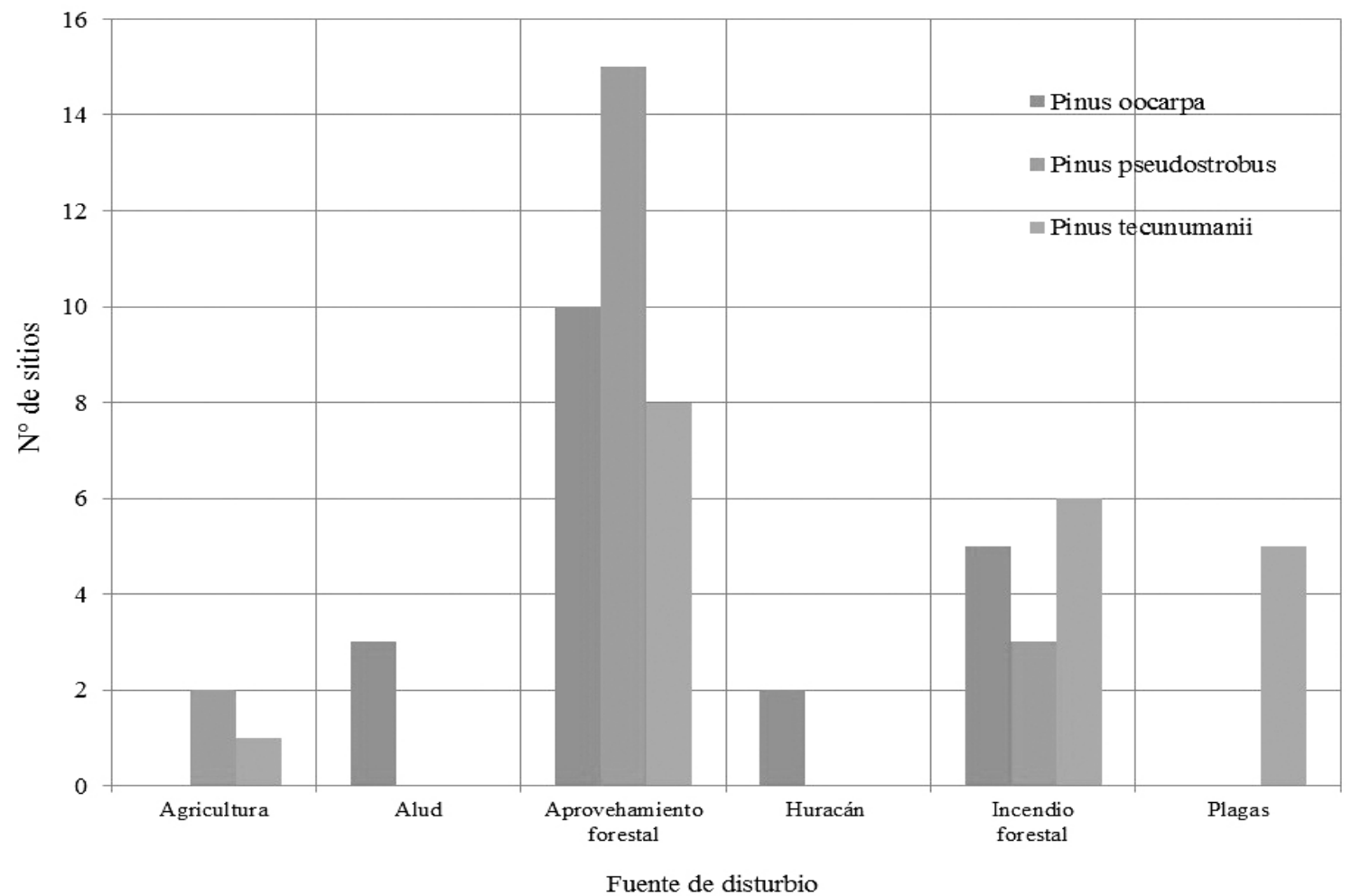

Figura 3. Fuentes de disturbio identificados en espacios de borde y claro, en bosques de Pinus oocarpa, Pinus pseudostrobus y Pinus tecunumanii del altiplano occidental de Guatemala.

afectados por alud y huracán la regeneración establecida fue únicamente de $P$. oocarpa.

Las mayores densidades de regeneración natural (Figura 4), se registraron con P. oocarpa en sitios afectados por alud, y fue la única especie regenerada en esta clase de disturbio. En segunda instancia con $P$. pseudostrobus en sitios que fueron destinados a agricultura. P. tecunumanii registró la mayor densidad en sitios afectados por aprovechamiento forestal e incendios forestales. La mayor densidad de especies acompañantes a la regeneración natural de pinos, se presentó en sitios afectados por incendios y aprovechamiento forestal y fue $P$. pseudostrobus, el que presentó las mayores densidades. Entre la diversidad asociada se halló la chispa (Pteridium caudatum (L.) Maxon).

La variación de la densidad respecto a la distancia de la fuente de semilla o progenitores, el espacio de borde presentó el comportamiento de $\mathrm{J}$ invertida (Figura 5a), o sea disminuyó con el aumento de la distancia en las tres especies. En espacio de claro la distribución de regeneración natural manifestó un patrón variable de subpoblaciones (Figura $5 b$ ). La densidad en los primeros $10 \mathrm{~m}$ de los progenitores en espacio de borde y claro fue bajo, manifestando la menor densidad P. pseudostrobus, como un hallazgo en el estudio.

Las distancias de regeneración natural establecida para $P$. oocarpa fue de $65 \mathrm{~m}$, en borde y $160 \mathrm{~m}$, en claro; para P. pseudostrobus fue de $75 \mathrm{~m}$, en borde y $175 \mathrm{~m}$, en claro; y para $P$. tecunumanii fue de $70 \mathrm{~m}$, en borde y $170 \mathrm{~m}$, en claro. Para los árboles padres de P. pseudostrobus son los de mayor porte de las tres especies, estimando una altura promedio de 24-30 $\mathrm{m}$ y densidades en área basal de hasta $28 \mathrm{~m}^{2} / \mathrm{ha}$, y la ocupación sitios en laderas de montaña con altas pendientes. P. oocarpa es de menor porte 15-20 $\mathrm{m}$ y densidades de área basal de hasta $18 \mathrm{~m}^{2} / \mathrm{ha}$, ocupando sitios de pie de monte y valles montanos en el área de estudio.

Las mayores densidades se presentaron en exposición norte para las tres especies y los mayores valores corresponden a P. oocarpa. La exposición Este fue favorable para $P$. oocarpa y $P$. tecunumanii y la Oeste favorable para $P$. pseudostrobus. Las exposiciones norte, este, sur y cenital fueron favorables para $P$. tecunumanii. La densidad de regeneración fue muy limitada para $P$. pseudostrobus para las exposiciones Cenital, Sur y Este (Figura 6). 


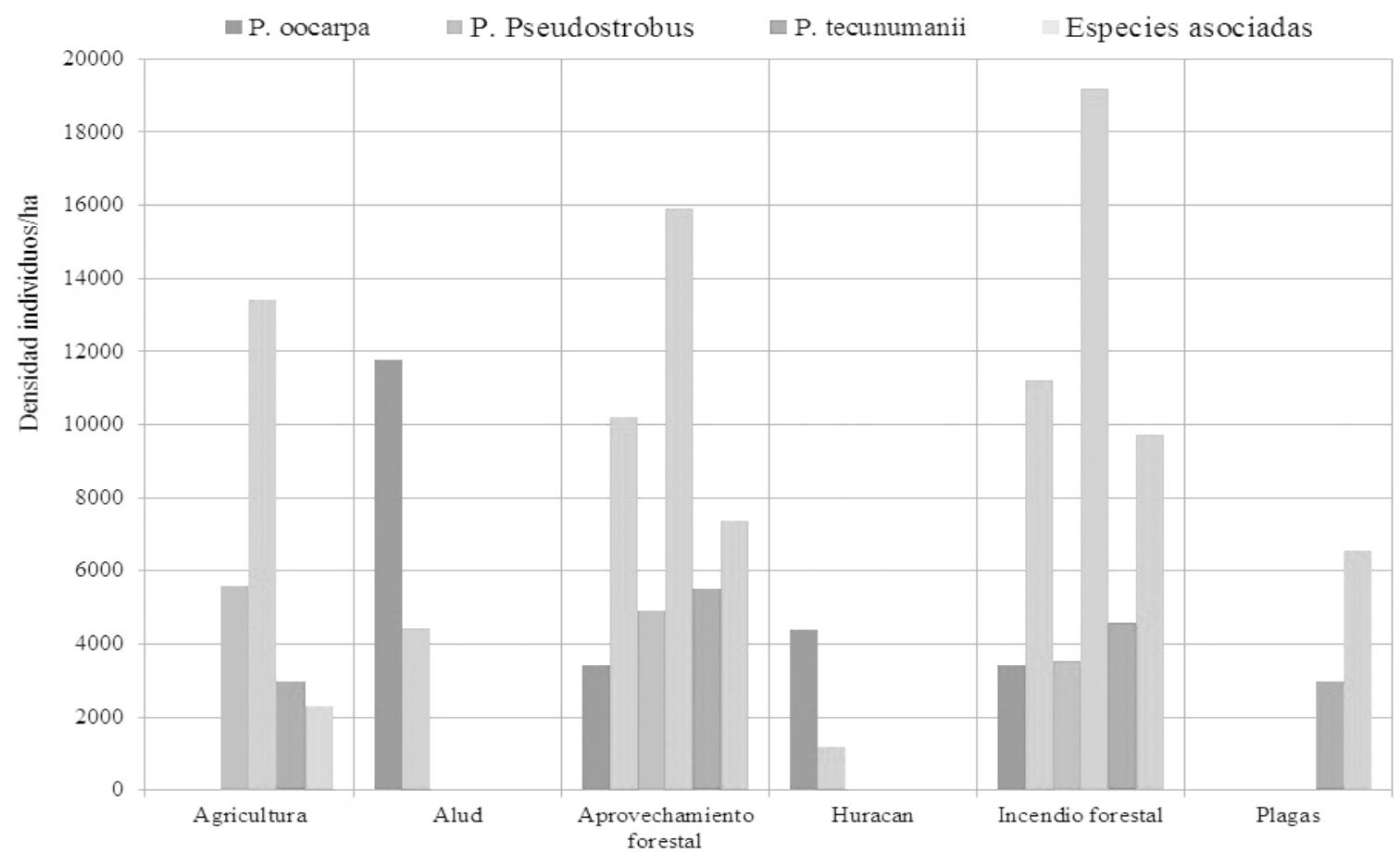

Fuente de disturbio

Figura 4. Comportamiento de la densidad de regeneración natural de Pinus oocarpa, Pinus pseudostrobus y Pinus tecunumanii por fuente de disturbio y especies asociadas.
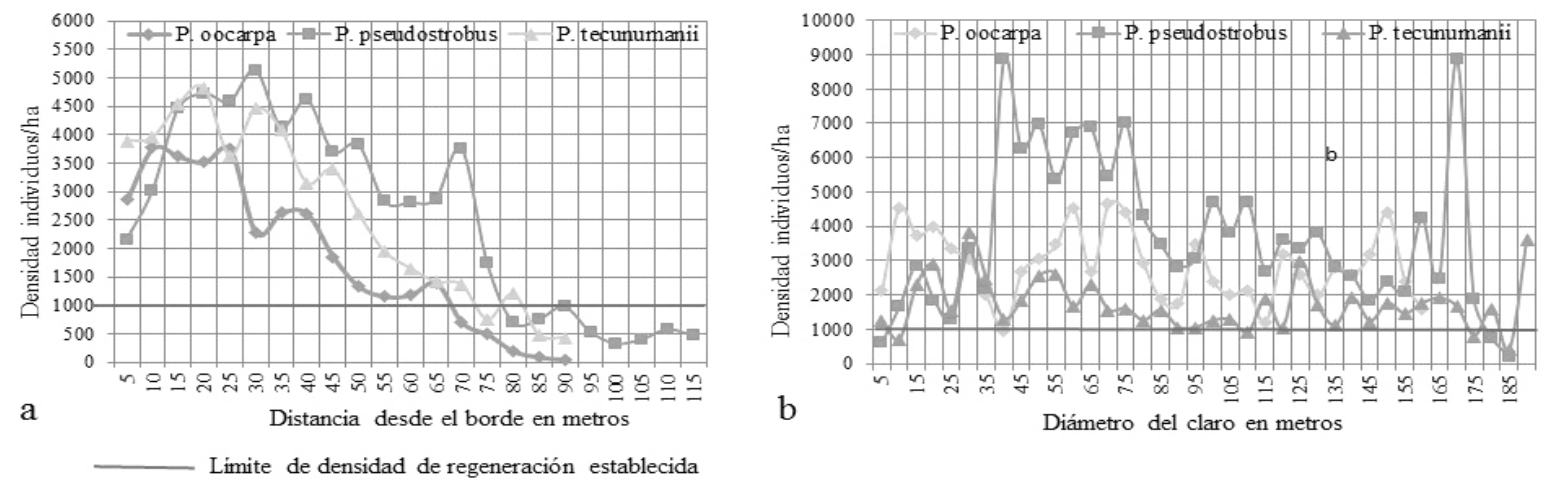

Figura 5. Comportamiento de la densidad de regeneración natural respecto de la fuente semillera y distancia de regeneración establecida, en espacios de (a) borde y (b) claros, en bosques de coníferas del altiplano occidental de Guatemala. 


\section{Discusión}

Se evaluaron 60 sitios con presencia de regeneración natural establecida, consecuencia de disturbios (Figura 3), resultado de la interacción de factores ambientales y antrópicos, que se congregan en dos grupos: los naturales o intrínsecos que se vinculan al clima, al suelo, al relieve, la disponibilidad de semilla y la competencia, y los antrópicos relacionados con el aprovechamiento y uso del suelo (Rebottaro \& Cabrelli, 2011; Rodríguez-García, Juez, Guerra, \& Bravo, 2007; Tiscar-Oliver, 2007).

El 16\% de sitios evaluados, pertenecen a factores naturales que favorecieron el establecimiento de regeneración de $P$. oocarpa por medio de alud y huracán, y P. tecunumanii por ataque plagas forestales al arbolado adulto. Los referidos disturbios y las especies establecidas, se enmarcan dentro del fenómeno de disturbios a pequeña escala que favorecen a las especies tolerantes a la sombra y los de gran escala favorecen a las especies intolerantes a la sombra (Habrouk, 2001), y a su condición de especies anemócoras, que son invasoras típicas de ambientes abiertos (Giorgis \& Tecco, 2014).

Y el $84 \%$ de los sitios evaluados con regeneración natural, corresponden a los factores antrópicos de extracción forestal, incendios y agricultura. La extracción forestal genera apertura de dosel, que atiende a las demandas de luz para el establecimiento de regeneración natural. Se reporta en estudios de coníferas, entre ellas la especie Pinus elliottii Engelm., que presentó mejor crecimiento en claros con ancho de 1.1 de la altura del arbolado con orientación este-oeste y un mejor establecimiento en claros con ancho de 0.8 y 1.1 de la altura del arbolado, independientemente de la orientación y un ingreso de radiación relativa mayor a $65 \%$ (Rebottaro \& Cabrelli, 2011). Además la perturbación del suelo por la actividad antrópica a cierta profundidad, influye en la llegada y el establecimiento de las especies a partir

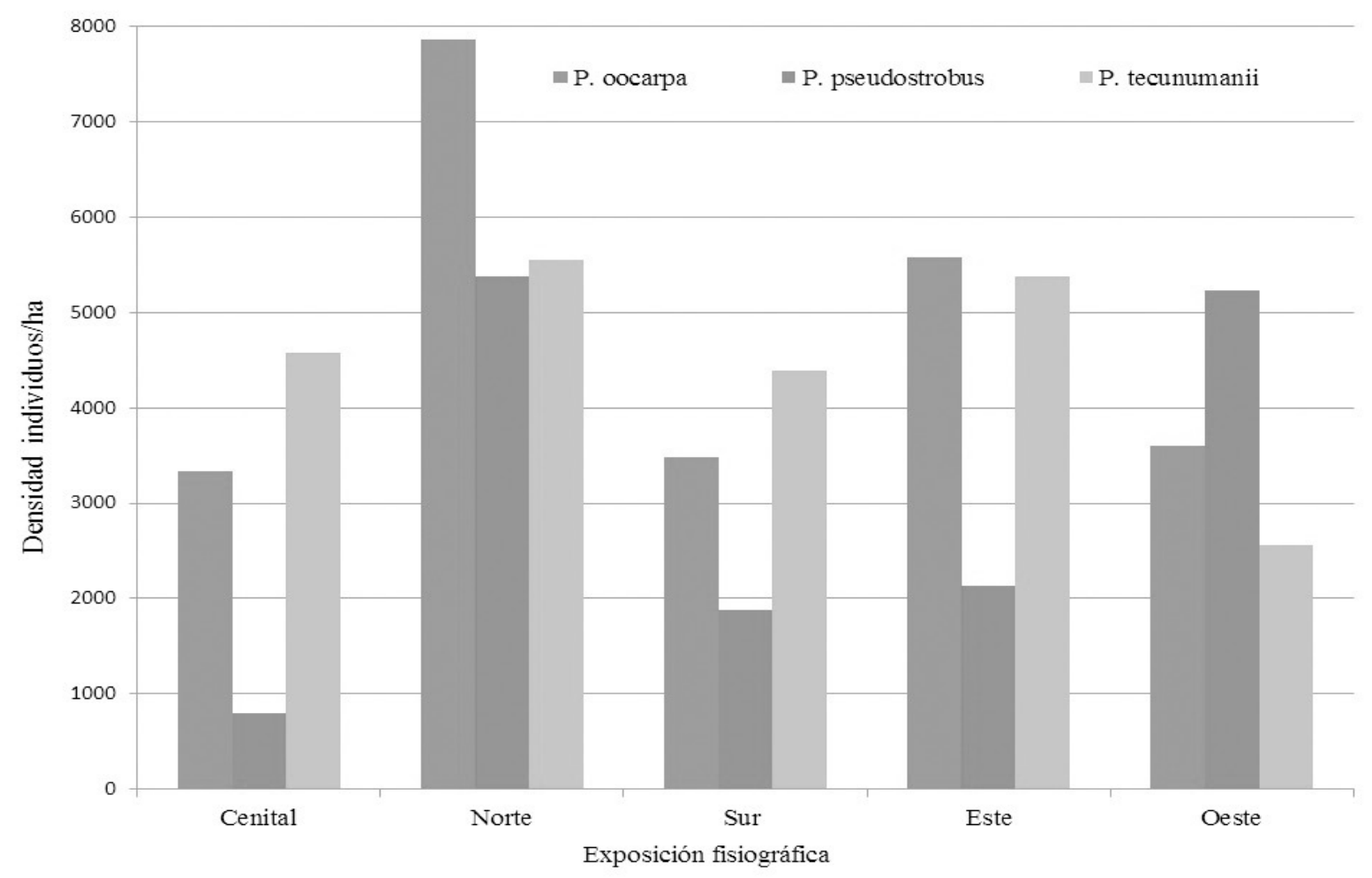

Figura 6. Tendencia de la densidad de regeneración de pino por exposición fisiográfica, en bosques de coníferas del altiplano occidental de Guatemala. 
del banco de semillas (Carrillo-Anzures, Vera-Casti1lo, Magaña-Torres, Guldin, \& Guries, 2009; Giorgis $\&$ Tecco, 2014).

Según lo expuesto, se infiere que la extracción forestal es un fenómeno que permite establecer regeneración natural de manera exitosa. También se reporta que incendios tienen como resultado positivo el incremento de la regeneración forestal (Arévalo et al., 2014); y han generado estrategias adaptativas como la corteza gruesa, la capacidad de auto poda y la recuperación de copas parcialmente quemadas en $P$. pseudostrobus (Rodriguez-Trejo \& Fulé, 2003). Lo citado permite explicar la respuesta de establecimiento exitoso de las tres especies en áreas afectadas por incendios y extracción forestal.

El modelo de J invertida (Figura 5a), como comportamiento de la variación de la densidad en espacio de borde, se relaciona con la distribución de las semillas, que genera descendencia agrupada cerca del progenitor y las colas alargadas de distribución a grandes distancias, que a la vez son expresiones de procesos de dispersión poco frecuentes (Clarck et al., 1998; Stoyan \& Wagner, 2001). Este comportamiento es altamente dependiente de la altura de los árboles y la dirección de los vientos dominantes, factor ambiental que influye en el comportamiento de la disminución de la densidad de semillas al aumentar la distancia al árbol madre o progenitor (González-Martínez \& Bravo, 1999; Kimmins, 1997).

Un modelo de subpoblaciones variables (Figura $5 b)$, se identificó en espacio de claro, con densidades extremas que variaron de 200 a 9,000 individuos/ha. Las subpoblaciones se presentaron en el centro de los claros, principalmente para las especies de P. pseudostrobus y $P$. oocarpa. Este modelo posiblemente se relacione con la disponibilidad y viabilidad de semilla. Para inferir lo expuesto, vale citar que en la colonización de espacios generados después de una perturbación, influye el banco de semillas (Carrillo-Anzures et al., 2009), y que la emergencia de las plántulas es influida positivamente por los claros o aperturas de dosel para Abies guatemalensis Rehder., en el occidente de Guatemala (Strandby, Prado, Sørensen, \& Kollman, 2006).

Además ambos modelos se relacionan con la distribución espacial de la regeneración natural, que replica el patrón definido por la dispersión de las semillas, con alto reclutamiento de individuos cerca de los progenitores, que pueden ser árboles o masa forestal circundante o en borde y una disminución logarítmica del número de individuos a medida que aumenta la dis- tancia a los progenitores (Sarasola et al., 2006), lo cual se relaciona con altas velocidades de viento (Greene \& Johnson, 1989).

La baja densidad de regeneración en los primeros $10 \mathrm{~m}$, cercanos a los progenitores en ambos espacios y acentuado en $P$. pseudostrobus se debe al sombreo. El crecimiento de la regeneración natural es afectado durante el proceso de establecimiento, por la sombra de la copa y los efectos adversos de los árboles progenitores y residuales (Chacón et al., 1998; Valkonen, 2000). En el estudio del efecto de la masa forestal remanente sobre la regeneración de Pinus sylvestris L., se estimó un efecto significativo sobre el crecimiento de las plántulas a una distancia de 3.5 a $5 \mathrm{~m}$ desde la base de los árboles padre (Valkonen, 2000). De lo anterior se infiere que la baja densidad más acentuada en P. pseudostrobus (Figura $5 \mathrm{a}, \mathrm{b}$ ), se debe a que es una especie caracterizada por sus altos requerimientos lumínicos al prosperar su establecimiento en sitios con apertura total de dosel (Alanís-Rodríguez et al., 2010; Negreros-Castillo \& Snook, 1884). Además se ha establecido que la reducción de crecimiento de la regeneración natural de pinos, se relaciona con la acción alelopática de los propios árboles progenitores a nivel radical y aéreo (Valkonen, 2000). También se ha determinado la influencia alelopática de la chispa $(P$. aquilinum (L.) Kuhn) en coníferas del norte de Idaho (Ferguson \& Boyd, 1988). La presencia y asocio de (P. caudatum) a la regeneración de P. pseudostrobus, permite inferir que el fenómeno de alelopatía por este helecho, sea uno de los factores que esté condicionando el comportamiento de la regeneración natural cerca de los árboles padre.

La densidad de especies asociadas a las tres especies de coníferas evaluadas, primero fue superior a la de la especie de pino asociada con excepción de los disturbios de alud y huracán (Figura 4). Segundo, las mayores densidades de especies asociadas a los pinos, se presentaron en los sitios regenerados afectados por extracción forestal e incendios. El comportamiento expuesto, atiende a hallazgos de que la distribución espacial de pies regenerados depende de los factores ambientales relacionados con la masa forestal, vegetación asociada y sus interacciones (Rodríguez-García et al., 2007), también con la intensidad, la calidad del espectro y la variación espacial de régimen lumínico (Emborg, 1998; González, 2013). Se ha reportado que P. sylvestris puede ser favorecido en su primer desarrollo por una ligera cubierta (González-Martínez \& Bravo, 1999), y en Pinus pinaster L., se ha observado una disminución 
de la regeneración de 7,000 a 4,000 pies/ha, al aumentar la cobertura de la vegetación acompañante del 50-75\% al $75-100 \%$, integrada por arbustos, hierbas y musgos (Rodríguez-García et al., 2007). En los sitios evaluados se presentan comportamientos similares de disminución de densidad de regeneración natural en $P$. tecunumanii y $P$. oocarpa (Figura 4) y un aumento de la densidad de las especies acompañantes en sitios afectados por incendios y aprovechamiento forestal.

Los sitios regenerados con P. pseudostrobus, con densidades comprendidas entre 3,500-5,500 pies/ha, reportaron las mayores densidades de especies asociadas (Figura 4). Este comportamiento se relaciona con diversos estudios en ambientes mediterráneos, que han demostrado que los arbustos mejoran la supervivencia de las plántulas de pino como consecuencia de una interacción de facilitación, siendo patente la influencia que la vegetación acompañante ejerce en el número de pies viables (Rodríguez-García et al., 2007; Tiscar-Oliver, 2007), aunque siempre compiten por su sobrevivencia con las especies herbáceas, matorrales y leñosas (González \& Bravo, 1999).

El alcance de dispersión de regeneración natural en espacio de borde varío de 90 a $115 \mathrm{~m}$ y en espacio de claro varío de 155 a $190 \mathrm{~m}$. Se reporta como límite del alcance de dispersión $50 \mathrm{~m}$ y un pequeño porcentaje de regeneración natural, alcanza distancias considerablemente mayores, incluso kilómetros de la fuente de origen, favorecido por las semillas que son ligeras y por ocurrencia de fuentes vientos (Ledgard, 2001). De acuerdo a este hallazgo, la dispersión de las tres especies para espacios de borde y de claro es superior, reportando las mayores distancias en ambos espacios P. pseudostrobus y luego P. tecunumanii.

La distancia de regeneración natural establecida respecto a la fuente de semilla, varió de 65 a $75 \mathrm{~m}$ para espacio de borde, y de 160 a $175 \mathrm{~m}$ para espacio de claro. Se reporta que entre el 85 al $90 \%$ de las semillas de Pinus sp., caen en los primeros 50 a $60 \mathrm{~m}$ de la fuente de semilla y que la distancia de establecimiento de regeneración natural de Pinus ponderosa Douglas ex C. Lawson, fue de 20 a $50 \mathrm{~m}$ y de Pinus contorta Dougl. ex. Loud., fue 50 a $100 \mathrm{~m}$, a partir del borde de la fuente de semilla en la región Andino-Patagónica (Sarasola et al., 2006). Con estos datos se infiere que las tres especies de pinos evaluados manifiestan un alto potencial de dispersión al superar los 50 y 100 m respectivamente. Para especies de luz, densidades entre 2,000 a 2,500 pies/ha se consideran exitosas (Serrada, 2003), al respecto las tres especies evaluadas cumplen con estos criterios de densidad, y las mayores densidades de regeneración las presenta $P$. pseudostrobus para bordes y claros, luego $P$. tecunumanii para espacios de borde y $P$. oocarpa para espacios de claro.

La mayor densidad y distancia de dispersión la presentó $P$. pseudostrobus para ambos tipos de espacio y la menor densidad y distancia de dispersión $P$. oocarpa (Figura 5), lo cual se relaciona con el porte en altura y densidad en área basal de ambas especies. Estos son algunos de los factores que explican en parte la distancia de dispersión y las densidades reportadas.

Respecto de la relación entre la densidad de regeneración natural y la exposición fisiográfica, la exposición norte es la más favorable y las menos favorables son las exposiciones sur y cenital. Mientras que para el establecimiento de regeneración natural para las tres especies evaluadas; este comportamiento es similar al observado en el monte Cabeza de Hierro de Madrid, donde se ha detectado la preferencia de la regeneración natural por las exposiciones norte (Rubio, 1987; Mori1lo, 1987; Santos, 1987 citados por González-Martínez \& Bravo, 1999).

P. pseudostrobus manifiesta mayor preferencia por las exposiciones norte y oeste que son las que reciben menos horas luz. P. oocarpa prospera mejor en las exposiciones norte y este. P. tecunumanii prospera bien en las exposiciones norte, este, cenital y sur, caracterizándola como la especie con mayor flexibilidad de adaptación de las tres especies evaluadas. La variación espacial del régimen lumínico es uno de los factores abióticos más citados que se relacionan con el establecimiento de regeneración natural de especies arbóreas forestales (Emborg, 1998). Además afecta la tasa de crecimiento que también depende de la orientación (González-Martínez \& Bravo, 1999). Estos hallazgos explican en parte el comportamiento de la regeneración natural de las tres especies en las diversas exposiciones en los sitios evaluados en el altiplano occidental de Guatemala.

\section{Agradecimientos}

Se agradece la cofinanciación por la Dirección General de Investigación de Universidad de San Carlos de Guatemala (Digi), dentro del Programa Universitario de Investigación en Recursos Naturales (Inf-2009-53), el apoyo del Ing. Saúl Guerra por el acompañamiento y motivación para investigar, al personal técnico y secretarial de la Región VII y Sub Región VII-2 de Inab al facilitar expedientes de aprovechamientos forestales; 
al personal técnico de la Oficina Forestal Municipal de Totonicapán y al Programa Mi Bosque de Care GTM por el apoyo al Programa de Ingeniería Forestal de $\mathrm{Cu}$ noroc, que permitió identificar el tema de investigación, al facilitar el acceso a los bosques de la Sierra María Tecún.

\section{Referencias}

Alanís-Rodríguez, E., Jiménez-Pérez, J., Pando-Moreno, M., Aguirre-Calderón, O. A., Treviño-Garza, E. J., \& García-Galindo, P. C. (2010). Efecto de la restauración ecológica post-incendio en la diversidad arbórea del Parque Ecológico Chipinque, México. Madera y Bosques, 16(4), 39-54.

Arévalo, J. R., Fernández-Lugo, S., Naranjo-Cigala, A., Salas, M., Ruíz, R., Ramos, R. \& Moreno, M. (2014). Post-fire recovery of an endemic Canarian pine forest. International Journal of Wildland Fire, 23(3), 403-409.

Carrillo-Anzures, F., Vera-Castillo, G., Magaña-Torres, O. S., Guldin, J. M., \& Guries, R. P. (2009). Seeds storend in the floor in a natural stand of Pinus montezumae Lamb. Ciencia Forestal, 34(106), 41-60.

Chacón, J. M., Velásquez, A., \& Musalem, M. A. (1998). Comportamiento de la repoblación natural de Pinus arizonica Engelm. bajo diferentes coberturas. Madera y Bosques, 4(2), 39-44.

Clarck, J. S., Fastie, C., Hurtt, G., Jackson, S. T., Johnson, C., King, G. A., ...Wickoff, P. (1998). Reid's paradox of rapid plan migration. Dispersal theory and interpretation of paleoecological records. Bioscience, 48(1), 13-24.

Consejo Nacional de Áreas Protegidas. (2009). Lista de especies amenazadas de Guatemala-LEA-y listado de especies de flora y fauna silvestres CITES de Guatemala ( $2^{\mathrm{a}}$ ed.). Guatemala: Autor.

Emborg, J. (1998). Understorey light conditions and regeneration with respect to the structural dynamics of a near-natural temperate deciduos forest in Denmark. Forest Ecology and Management, 106 (Issues 2-3), 83-95.

Ferguson, D. E., \& Boyd, R. J. (1988). Bracken fern inhibition of conifer regeneration in northern Idaho (Research paper INT-388). Ogden,UT, Idaho: Departament of Agriculture, Forest Service,
Intermountain Research Station. Recuperado de https://archive.org/details/brackenferninhib388ferg Giorgis, M. A., \& Tecco, P. A. (2014). Árboles y arbustos invasores de la Provincia de Córdoba (Argentina): Una contribución a la sistematización de bases de datos globales. Boletín de la Sociedad Argentina de Botánica, 49(4), 581-603.

Godínez, S. M., Rodríguez, F. A., Camposeco, J. I., \& López, N. P. (2010). Evaluación de la regeneración natural de tres especies coniferas en áreas de distribución natural en el altiplano occidental de Guatemala (Inf-2009-053). Guatemala: Universidad de San Carlos de Guatemala, Dirección General de Investigación, Centro Universitario de Nor Occidente.

González, E. (2013). Patrones espacio-temporales de dispersión y de regeneración del abeto (Abies alba Mill.) en los Pirineos Aragoneses (Tesis de maestría). Universidad Autónoma de Madrid, España. Recuperado de https://www.uam.es/ departamentos/ciencias/ecologia/Posgrado $\% 20$ en $\% 20$ Ecologia/Tesis\%20Leidas/Tesis\%20 Maestria\%20(TFM)/Leidas\%202013/TFM\%20 Ester\%20Gonzalez.pdf

González-Martínez, S. C., \& Bravo, F. (1999). Regeneración natural, establecimiento y primer desarrollo del pino silvestre (Pinus sylvestris L.). Palencia: Universidad de Valladolid, Departamento de Producción Vegetal y Silvopasticultura. Recuperado de http://www.inia.es/IASPF/1999/ Allue/15.S.C.GONZALEZ.pdf

Greene, D. F., \& Johnson, E. A. (1989). A model of wind dispersal of winged or plumed seeds. Ecological, 70(2), 339-347. doi: 10.2307/1937538

Greene, D. F., Messier, C., Asselin, H., \& Fortin, M.-J. (2002). The efect of light availability and basal area on cone production in Abies balsamea and Picea glauca. Canadian Journal of Botany, 80(4), 370377. doi: 10.1139/B02-020.

Habrouk, A. (2001). Regeneración natural y restauración de la zona afectada por el gran incendio del Bages y Bergueda de 1994 (Tesis de doctorado). Universidad Autónoma de Barcelona, España. Recuperado de http://www.tdx.cat/bitstream/ handle/10803/3647/ah1de4.pdf; jsessionid=29D3D4F78488253C10420399DFE715DE.tdx1?sequence $=1$ 
Hawley, R. C. \& Smith, D. M. (1982). Silvicultura práctica ( $2^{\mathrm{a}}$ ed.). Barcelona: Omega.

Instituto Nacional de Bosques. (2001). Manual para la elaboración de planes de manejo forestal en bosques de coniferas, (modelo centroamericano). Guatemala: Autor.

Kaufmann, M. R., Graham, R. T., Boyce Jr., D. A., Moir, W. H., Perry, L., Reynolds, R. T., ... Corn, P. S. (1994). An ecological basis for ecosystem management (General Technical Report RM-246) . Fort Collins, CO: Department of Agriculture, Forest Service, Rocky Mountain Forest and Range Experiment Station. Recuperado de http:// www.fs.fed.us/rm/pubs_rm/rm_gtr246.pdf

Kimmins, J. P. (1997). Forest ecology (2 $2^{\mathrm{a}}$ ed.). London: Prentice Hall.

Ledgard, N. (2001). The spread of lodgepone pine (Pinus contorta Dougl.) in New Zeland. Forest Ecology and Management, 141(2-3), 43-57.

Martínez, I., \& González-Taboada, F. (2009). See dispersal patterns in a temperate forest during a mast event: Performance of alternative dispersal kernels. Oecologia, 159, 389-400. doi: 10.1007/ s00442-008-1218-4.

Oliver, C., \& Larson, B. (1996). Forest stand dynamics. New York: McGraw-Hill.

Organización Internacional de Maderas Tropicales. (2002). Directrices de la OIMT organización Internacional de Maderas Tropicales para la restauración, ordenación y rehabilitación de bosques tropicales secundarios degradados (Serie de políticas Forestales No. 13). Yokohama, Japón: Autor.

Programa de las Naciones Unidas para el Desarrollo PNUD. (2014). Sinopsis del desarrollo humano en Guatemala. Guatemala: Autor.

Rebottaro, S. L., \& Cabrelli, D. A. (2011). Regeneración natural de Pinus elliottii en claros silvícolas: Dinámica poblacional durante siete años. Madera y Bosques, 17(1), 49-70.

Rodríguez-García, E., Juez, L., Guerra, B., \& Bravo, F. (2007). Análisis de la regeneración natural de Pinus pinaster Ait. en los arenales de AlmazánBayubas (Soria, España). Sistemas y Recursos Forestales, 16(1), 25-38.
Rodríguez-Trejo, D. A. \& Fulé, P. Z. (2003). Fire ecology of Mexican pines and a fire management proposal. International Journal of Wildland Fire, 12, 23-37.

Sarasola, M. M, Rusch, V. E., Schlichter, T. M., \& Ghersa, C. M. (2006). Invasión de coníferas forestales en áreas de estepa y bosques de ciprés de la cordillera en la Región Andino Patagónica. Ecología Austral, 16, 143-156.

Serrada, R. (2003). Regeneración natural: Situaciones, concepto, factores y evaluación. Cuaderno Sociedad Española de Ciencias Forestales, 15, 11-15.

Negreros-Castillo, P. \& Snook, L. (1984). Análisis del efecto de la intensidad de corta sobre la regeneración natural de pinos. Ciencia Forestal, 47(9), 48-61.

Stoyan, D., \& Wagner, S. (2001). Estimating the fruit dispersión of anemocorus forest trees. Ecological Modelling, 145, 35-47.

Strandby, U., Prado, J., Sørensen, M., \& Kollmann, J. (2006). Conservation and utilisation of Abies guatemalensis Rehder (Pinaceae) - an endangered endemic conifer in Central America. Biodiversity and Conservation, 15, 3131-3151. doi: 10.1007/ s10531-005-5405-x.

Tiscar-Oliver, P. A. (2007). Dinámica de regeneración de Pinus nigra subsp. salzmannii al sur de su área de distribución: Etapas, procesos y factores implicados. Sistemas y Recursos Forestales, 16(2), 24-135.

Valkonen, S. (2000). Effect of retained scots pine trees on regeneration, growtn, form, and yield of forest stands. Sistemas y Recursos Forestales, (1), 121-145.

van Mantgem, P. J., Stephenson, N. L., \& Keeley, J. E. (2006). Forest reproduction along a climatic gradient in the Sierra Nevada, California. Forest Ecology and Management, 225(1), 391-399. doi: 10.1016/j.foreco.2006.01.015

White, P. S., \& Pickett, S. T. (1985). Natural Disturbance and patch dynamics: An introduction. S. T. Pickett \& P. S. White (Eds.), The Ecology of natural disturbance and patch dynamics (pp. 3-13). New York: Academic Press 